\title{
Towards natural handshakes for social robots: human-aware hand grasps using tactile sensors
}

https://doi.org/10.1515/pjbr-2018-0017

Received November 30, 2017; accepted June 23, 2018

Abstract: Handshaking is a fundamental part of human physical interaction that is transversal to various cultural backgrounds. It is also a very challenging task in the field of Physical Human-Robot Interaction (pHRI), requiring compliant force control in order to plan the arm's motion and for a confident, but at the same time pleasant grasp of the human user's hand. In this paper, we focus on the study of the hand grip strength for comfortable handshakes and perform three sets of physical interaction experiments between twenty human subjects in the first experiment, thirty-five human subjects in the second one, and thirty-eight human subjects in the third one. Tests are made with a social robot whose hands are instrumented with tactile sensors that provide skin-like sensation. From these experiments, we: (i) learn the preferred grip closure according to each user group; (ii) analyze the tactile feedback provided by the sensors for each closure; (iii) develop and evaluate the hand grip controller based on previous data. In addition to the robot-human interactions, we also learn about the robot executed handshake interactions with inanimate objects, in order to detect if it is shaking hands with a human or an inanimate object. This work adds physical human-robot interaction to the repertory of social skills of our robot, fulfilling a demand previously identified by many users of the robot.

Keywords: human-robot interaction, physical humanrobot interaction, tactile sensing, handshake, handshake recognition

\footnotetext{
^Corresponding Author: João Avelino: Institute for Systems and Robotics, Instituto Superior Técnico, University of Lisbon; E-mail: javelino@isr.tecnico.ulisboa.pt

Tiago Paulino, Carlos Cardoso: Institute for Systems and Robotics, Instituto Superior Técnico, University of Lisbon;

E-mail: \{tiago.paulino, carlos.cardoso\} @tecnico.ulisboa.pt

Ricardo Nunes, Plinio Moreno, Alexandre Bernardino: Institute for Systems and Robotics, Instituto Superior Técnico, University of Lisbon; E-mail: \{rnunes, plinio, alex\} @isr.tecnico.ulisboa.pt
}

\section{Introduction}

The handshake between humans is a social ritual that has various connotations according to the cultural background and can convey trust, recognition, and equality between the participants. The initial steps of social interactions between humans usually include handshaking, so in humanoid robots the capability of performing handshaking actions and the ability to detect them properly may help interaction in many human-robot scenarios. Handshaking is a very complex interaction that includes intricate sensorimotor skills such as force-compliance, tactile feedback, and gaze synchronization. Force compliance provides the skills for the correct motion of the wrist for leading and following the human handshake movement. Tactile feedback can provide the skills for the correct motion of the finger limbs for an adequate pressure value of the handshake. Gaze synchronization is a clue that conveys the signal for starting/ending the handshake. Providing the skills mentioned above to a humanoid robot is a very complicated task that is currently limited mainly by the development of tactile sensing, where the development of materials that can sense properly and provide comfort are one of the main problems. A handshake is defined as a grasp between two people's hands that is usually accompanied by short up-and-down movement of the grasping hands. We define comfort as "the strength that conveys an adequate handshake" and only focus on the grasping part of the handshake. In this work we address tactile sensing problems in the context of handshaking between a person and a humanoid robot, considering two scientific questions: (i) Does the hand-shaped end-effector of our humanoid robot provide a comfortable sensation in terms of force and touch interaction during the handshake? and (ii) Is the robot capable of detecting if it is grasping a human hand or another inanimate object during handshake interactions? These questions address the problem of designing a "comfortable" human-robot hand grip following a user-centered design approach since user feedback and preferences are incorporated in the design of a "comfortable" handshake. In Figure 1 we can see a 


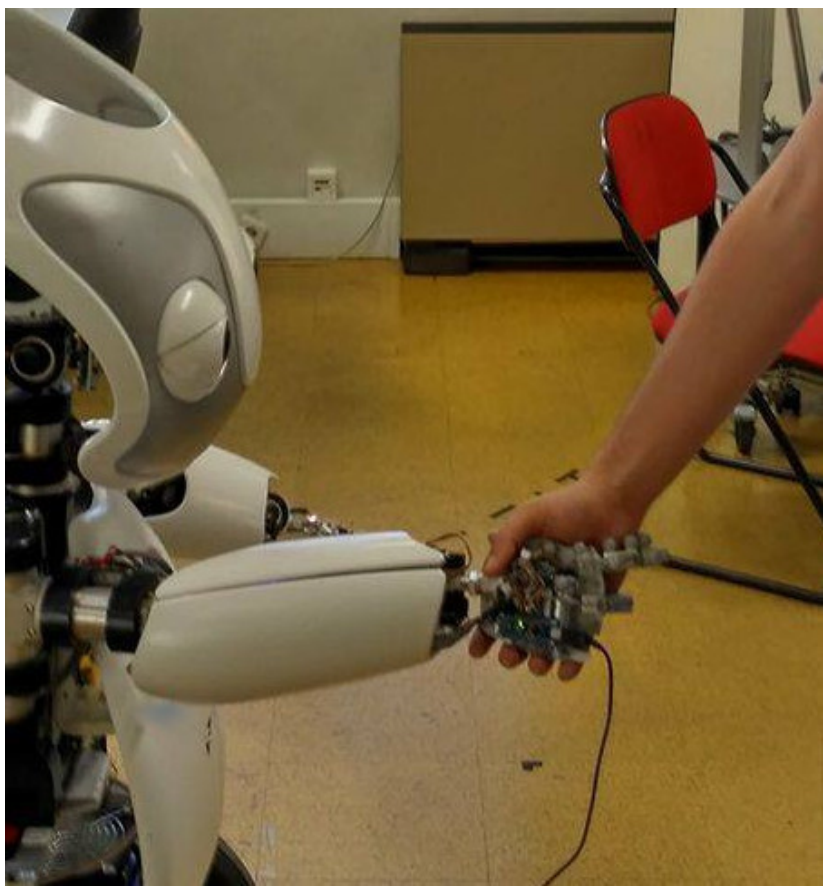

Figure 1: The robot Vizzy performing a handshake with one of the subjects.

handshake between a human and the robot Vizzy. The four fingers of the robot's hand are instrumented with a total of 15 tactile sensors. The tactile sensors' configuration yields the force distribution on several contact points, rather than solely the total force of the hand grip. This is important since even if we have a total hand grip force that could be considered acceptable, it could cause harm to the person's hand, if it is focused on a single point instead of being distributed. The sensors have two main functions: (i) measure the forces being exerted at the points of contact and (ii) provide compliance and a more human-like touch feedback to the user.

Recent developments on tactile sensors provide both a comfortable contact for handshaking and an accurate measurement of the force [1]. The sensors provide an estimation of the force from the changes in the magnetic field, considering three main elements: (i) 3 dimensional hall effect sensor, (ii) magnet and (iii) a silicone cover for the magnet (see Figure 2). The changes in magnetic field due to the deformation of the silicone part are mapped onto 3 dimensional forces, which provide the tactile perception to the silicone cover. Since silicone is one of the materials that is being studied for providing a skin-like sensation [2], we expect that people will feel comfortable during handshaking from the touch interaction point of view. However, the force exerted by the robot will play a fundamental role. Thus, to find the most comfortable grip we performed two studies: (i) a pilot study with predefined finger configurations (Section 4) from which we intend to gather initial insights (regarding technical issues and experimental design), and (ii) a study where the users had the control of the robot's fingers (Section 6).

In addition to the quality of the handshake, the basic perceptual skill of a robotic handshake is to distinguish between grasping an object and a human hand from tactile feedback. This skill is the basic building component to avoid awkward situations that could originate from totally autonomous handshaking, hence allowing a robot to take corrective actions in case of disengagement and deceiving interactions. Furthermore, it is crucial that robots are able to distinguish whether they are involved in a handshake or an object handover interaction. We gather the magnetic field and force data from the sensors during handshaking interactions with persons and inanimate objects, feeding the data to a supervised machine learning binary classifier. On subsequent interactions with objects, the classifier is able to detect if the interaction was with a hand or with an inanimate object (Section 5).

In Section 2 we perform a literature review of developed works related to the subject of human-robot handshaking. Then, we describe our robot's hand and the used sensors in Section 3. The studies in this work can be divided into two: parts i) a human subject research of the preferable handshake grip force (Sections 4, 6 and 7) and ii) training a classifier to detect if the handshake was successful (Section 5). Each Section contains their respective methodologies and results. Then, in Section 8 we make some conclusions about our work and propose several ideas for future work.

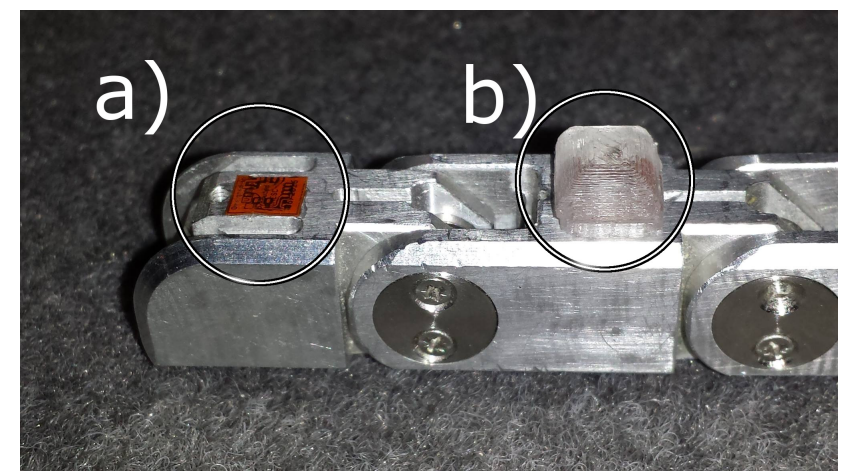

Figure 2: Tactile sensors mounted on the robot's fingers. On the left (a): Hall effect sensor. On the right (b): Full tactile sensor with the soft elastometer body and magnet. 


\section{Related work}

Physical Human-Robot Interaction (pHRI) is a field whose origins date back to the 90 s and 2000s [3]. It is nowadays receiving increased attention due to recent developments in haptic sensors [1] as well as applications in either social robotics [4] or industrial environments [5]. Handshaking is one of the subjects studied in pHRI and is starting to receive interest by several authors. The majority of works on human-robot handshakes focus on the planning and the shake motion $[6,7]$ of the robotic arm and mimicking a human's grasp $[8,9]$.

Several works study human-human handshakes as a basis for human-robot handshaking. In [10] a handshaking approach model is proposed based on the analysis of the position of the wrists and hands of the participants. This motion model is further expanded in [11] to take the start time in a handshake request into consideration. Similarly, [12] also studies gaze when requesting for a handshake in addition to the previous motion model. The lag between the start of the request in a handshake and the start of a response, as well as the motion model of the response preferred by humans, are discussed in $[13,14]$. [15] also studies the duration, strength of grip, vigor, and rhythmicity of handshakes between humans. They make use of six force sensors to study the strength of grip. Another study [16] focusing on arm motions during handshakes, proposes 3 models: tit-for-tat model, $\lambda$ model and IML-Shake model. The tit-for-tat model imitates previous human motions. The $\lambda$ model generates handshake motions based on biomechanical and physiological models of the handshake. The iML-Shake model uses a simple Machine Learning linear method to learn the movements from examples. These handshakes were tested using a Turing-Like Handshake Test to assess if the movements were human-like. The study concludes that the titfor-tat and iML-Shake models produce the most humanlike handshake movements.

In [17], a handshaking reactive robotic interface was developed. The designers of this solution took haptics into consideration for both mechanical design as well as controller design of the interface. To comfortably close the hand of the robot, the authors of this work measured the grasping force of humans, which was observed to have a median value of $25 \mathrm{~N}$ and a maximum value of $50 \mathrm{~N}$. The resulting interface consists in a four-fingered hand. It is also worth noting that during the user studies with the final interface, the authors noted that grasping forces applied by the subjects on the robot were different from those of human-human interaction. However, given the fact that a robotic hand has different pressure points than human hands, using the overall grasping force as a control reference might pose some comfort problems. This issue is covered in [18], showing that even with a lower overall grasping force, there are points in the robotic grasp where the pressure is much higher (and possibly more uncomfortable) than the one produced by a human hand.

Another interesting work [19], intends to create a model of tactile features to discriminate intrinsic characteristics of a person. They are able to recognize the gender of a person with a success rate of $77 \%$ and the extroversion with $62 \%$ success rate. For female participants, the mean sensor pressure on the sensors was of $25.8 \mathrm{kPa}$, with a standard deviation of $22.3 \mathrm{kPa}$. For males, it was of $29.4 \mathrm{kPa}$ and $16 \mathrm{kPa}$, respectively.

None of the above works, however, seem to employ a user-centered approach, where the handshake grasping is designed directly with user feedback. Moreover, to our knowledge, no attempts were made to discriminate between a fake and a real handshake. Works like [20], where the system is able to classify between several materials with tactile sensors, or [21, 22] that focus on object recognition make us believe that tactile features are rich enough for this matter.

\section{Robotic hand design}

The robotic platform used in this work is the robot Vizzy [23], that was designed as a human assistant for social interaction tasks. It has an anthropomorphic upper body with similar degrees of freedom and motion execution skills of a human and wheels on the lower body. Regarding its hands, the palm and finger sizes and number of joints are also similar to an adult person (although slightly bigger), but the hand has only four fingers capable of grasping objects. The thumb and index fingers are each actuated by a single motor, while the middle and ring fingers are coupled to one motor. The motor of a finger is coupled to a pulley, that pulls a fishing line string. The fishing line string is attached from the pulley to the last joint of the finger, such that the motion of one motor moves in an underactuated manner the three joints of each finger. Regarding the sensors, the thumb has three sensors and the rest of the fingers have four sensors each. The sensors are distributed as shown in Figure 3.

These tactile sensors are composed of a soft elastomer body with a small permanent magnet inside (Figure 2). Below the magnet, there is a magnetic field sensing element (i.e. Hall-effect sensor). When an external force is applied 


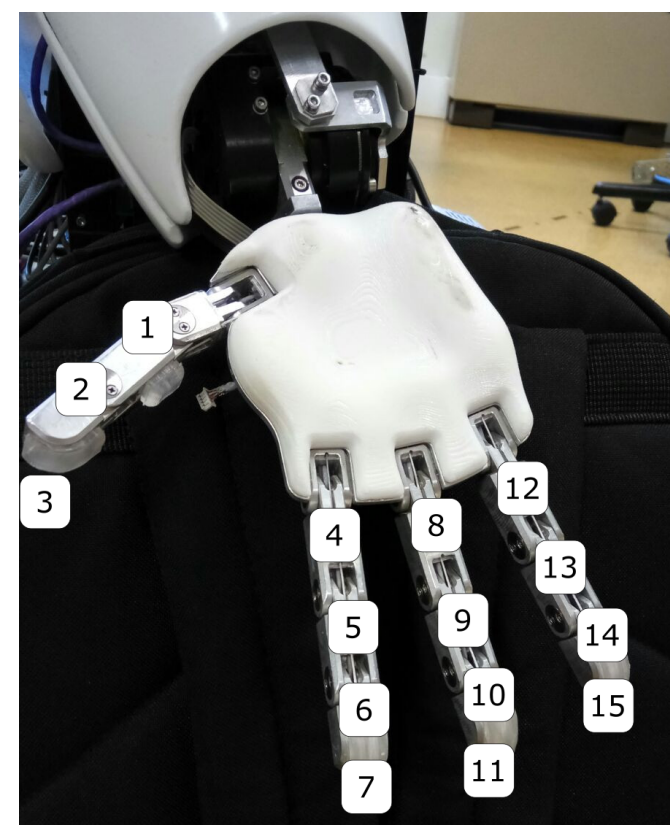

Figure 3: Indexes of the force sensors in our robot's hand. Sensors 12 to 15 are still on testing phase and will only be used in future work.

on the elastomer the relative magnet position changes and the Hall-effect sensor detects the magnetic field variation, which can be converted for the measurement of the applied force. An air gap is left between the elastomer and the magnetic sensor in order to increase the sensitivity for small forces. The use of a 3-axis Hall-effect sensor allows the detection of the magnetic field variations in the 3 axis, meaning the sensor is capable of measuring the force magnitude and direction in 3D. These sensors are capable to detect minimum forces in the order of $10 \mathrm{mN}$. More detailed information on the sensors' capabilities can be found in the work of Paulino et. al [1]. The hand design criteria included: (i) Similarity to a human's hand size and (ii) the execution of two types of object grasping: cylindrical and power grasp. It also includes a white palm cover (as seen in Figure 3) that was used in the studies presented in Section 6 and Section 7 as an improvement over the standard metallic palm. The motor control approaches considered in this work include (i) motor encoder position and (ii) total force magnitude per finger. Both the encoder and force controllers are implemented as a PID. For each motor in the hand, a PID controller was designed and implemented to maintain a force proportional to the sum of the forces sensed in all the corresponding finger sensors. This way each finger motor has independent control based on the force feedback from the sensors, that was used in the study in section 7 . Since the hand design did not consider handshake actions, we performed a user human- robot hand grip study for evaluating the plausibility of that kind of interaction. In the pilot study in Section 4 we make use of only three fingers: thumb, index and middle. The fourth finger was removed from the hand, being entirely absent from the handshake. In the study in Section 6 all fingers were used to perform the handshake, but since the sensors on the fourth finger are still in the test phase, the measured values were not recorded.

\section{Human-robot handshake: pilot study}

\subsection{Objectives}

The objectives of this pilot study are: (i) to gather an initial guess of user's preferences regarding human-robot handshake force distributions; (ii) to obtain insights regarding possible problems with the design of the experiment, and (iii) to obtain user feedback regarding the mechanical properties of the robot's hand and perceived comfort.

\subsection{User statistics}

The pilot experiment was performed on a population of 20 subjects, 13 male and 7 female, with ages from of 20 to 51 years old ( $\mu=32.95, \sigma=9.26)$. These subjects were researchers and staff from the authors' research group with different nationalities and cultures.

\subsection{Methodology}

In order to assess user's preferences regarding handshake grip strength, the pilot study consisted in a series of experiments with user and the robot. During these experiments, the people were asked to shake hands three times with the robot. The handshake started with an initial position of finger joints (controlled by three motors), followed by a timely closing of the fingers to the final position. The final motor positions are associated to the handshake strength label, having the largest motor positions with the label "strong", the lowest motor positions as "weak" and the intermediate motor positions as "medium". After the execution of the three handshakes we ask the participants to rank the handshake by their preference using three labels "bad", "average" and "good". The users are asked to rank the handshakes by considering mainly the strength that conveys an adequate handshake interaction. This means 
that the strength should be high enough to be engaged in the handshake, and at the same time low enough that does not make the person feel uncomfortable nor causes an injury. This means that handshakes with very low strength are ranked low as well as handshakes with very high strength. To avoid biased opinions due to eventual meetings between participants or their expectancy regarding the sequence of handshakes, the order of the three handshakes was counterbalanced. After performing the full experiment participants were also asked about their overall opinion about the trial.

\subsection{Data analysis}

Given the fact that different people have different hand sizes, analysis of handshake preferences based just on the final position of the fingers of the robot may not be meaningful. Since the same finger position would have a very large variability across different hand sizes, we analyzed the mean and variance of the forces measured by each sensor using two different ways of grouping: (i) The strength label from the final finger position and (ii) the preference level provided by the users. We collect the temporal sequence of the magnetic flux and force data from each tactile sensor during the handshake interactions, which is utilized in the hand detection study.

\subsection{Results and discussion}

To visualize the magnitude of the forces applied on the sensors for each movement, we plot in Figure 4 the means and variances of the sensor readings. As we can see, sensors number 3 and 11 (fingertips), and sensors 9, 10 (middle finger) make almost no contact with the human's hand. This is to be expected since the robot's hand is slightly larger than the average human's hand and the underactuated finger limbs move to different final configurations depending on the initial contact points. As expected, the variance in the most active sensors for each closing is large due to the different sizes of human hands.

The total force magnitudes presented in Table 1 correspond to the sum of forces at the contact points between the human hand and the tactile sensors. There is high area of contact (mainly in the palm of the robot hand) where the force is not measured which is the reason why the obtained values are under the expected total force of a human hand grip. The calculation of the hand grip force, however, was never the objective of the experiment. The goal is to study the most comfortable force distribution along the available

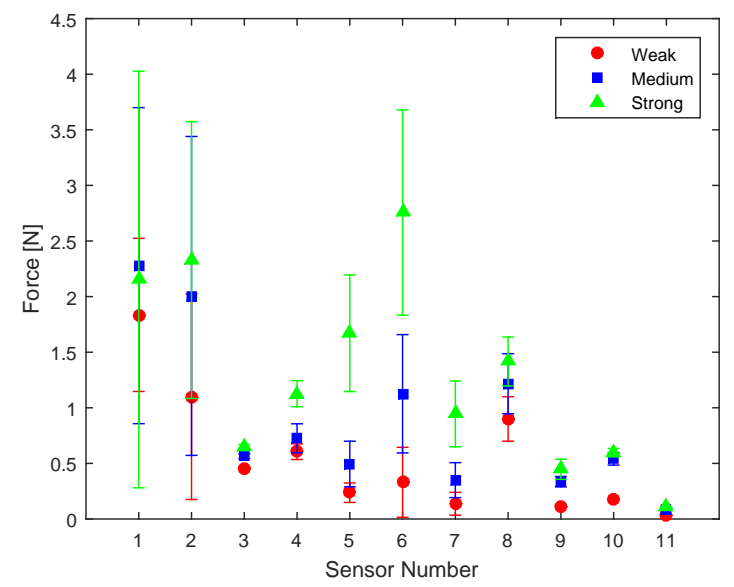

Figure 4: Average and variance of the force measured in each sensor for each hand grip action (best seen in color).

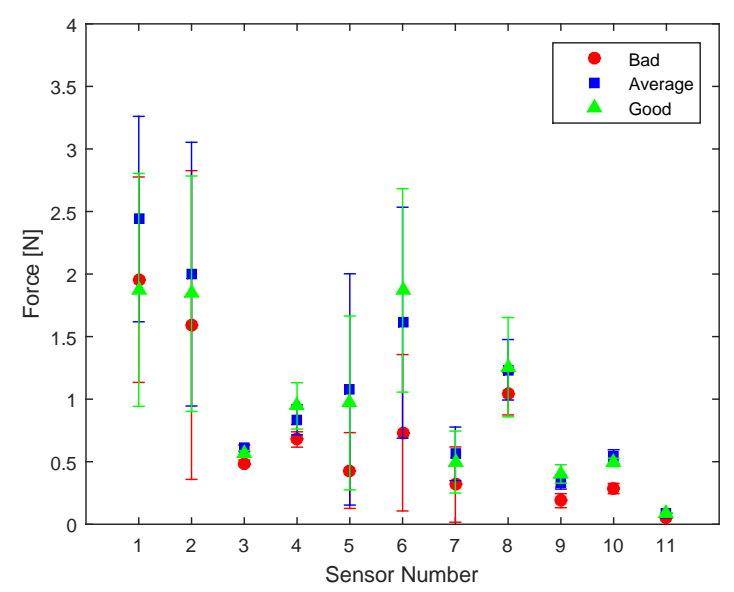

Figure 5: Average and variance of the force measured in each sensor according to the user preference (best seen in color).

sensors. Statistical information regarding this distribution is presented in Figure 5. We note that people preferred similar forces on sensors 1, 2, and 6 that represent the main contact points of the thumb and pointer fingers. These preliminary results give us an idea of force distributions that can be used as feedback for a handshake grip strength controller.

From the data collected we can observe that female subjects displayed a tendency to prefer (with no statistical significance) a slightly larger grip force than the male subjects (see Table 1 and Table 2). This can be explained by the 3 different grips being defined only by the final angular position of the encoders in the motors. The resulting force that is applied by the robot and felt by the human subject is produced by the elasticity and compliance of the artificial tendons and sensors in the robot hand. Consequently, 
the force felt by the human appears to be highly dependent on the shape and size of the human hand. We note that, for smaller hands, two of the hand grips have very low contact forces leading people to prefer the third hand grip, since there were no better options.

Table 1: Average sum of sensor forces by user feedback.

\begin{tabular}{c|ccc}
\hline \multicolumn{4}{c}{ Average force (N) } \\
\hline Grade & Bad & Average & Good \\
\hline Female & 6.79 & 7.96 & 11.41 \\
Male & 8.63 & 13.22 & 10.50 \\
Total & 7.76 & 11.33 & 10.81 \\
\hline
\end{tabular}

Table 2: Preferred handshake action.

\begin{tabular}{c|ccc}
\hline \multirow{4}{*}{} & \multicolumn{3}{c}{ Preferred handshake (\%) } \\
\cline { 2 - 4 } & Weak & Medium & Strong \\
\hline Female & 0.0 & 14.3 & 85.7 \\
Male & 15.4 & 46.1 & 38.5 \\
Total & 10.0 & 35.0 & 55.0 \\
\hline
\end{tabular}

In this experiment the preferred handshake was selected by 11 persons out of 20 (55\%) where the chance level was $33 \%$. A one-sample binomial test provides a p-value of 0.0552 , showing there is significance in the results presented. Nonetheless, we would like to point out that the population on this pilot study might not be representative of the general population.

\subsection{Qualitative feedback}

The feedback given by the human subjects shows that, despite the metallic hand, the silicon sensors give a very comfortable touch and grip. Many were surprised with a much more comfortable handshake in opposition to the initial expectations due to the robotic looks of the robot's hand. However, they had some constructive criticism regarding the thumb contacts. These were perceived as slightly stronger than the remaining fingers, thus needing adjustment. Regarding the execution of the handshake, people suggested that all the fingers should close at the same time and that the arm should execute the oscillatory motion of a handshake. Concerning the aesthetics and design of the hand, our subjects reported that the hand was larger than expected, and that the palm of the robot should have the same tactile feeling as the material of the sensors. Includ- ing tactile sensors in the palm would increase the comfort of the handshake and simultaneously provide added perceptual information to exploit. The absence of one finger in the hand of the robot was also noticed, and it was suggested that it should be used independently of its ability to measure forces or not.

\section{Handshake detection study}

\subsection{Objectives}

In this section we intend to develop and evaluate a classifier that can allow the robot to be aware of successful and unsuccessful handshakes. We intend to do this with tactile data from the robot's hand sensors.

\subsection{Methodology}

In this section we tackle the problem of detecting whether the robot has performed a handshake with a human hand or with an other type of (inanimate) object. In order to do so we exploit the information gathered by force sensors and employ a supervised machine learning approach, the K-Nearest Neighbors algorithm with Dynamic Time Warping [24], a method that achieves good results on the classification of time series in small datasets [25]. The reason for this choice is that the number of handshake samples gathered is not large enough to estimate the parameters of advanced learning algorithms such as deep neural networks, avoiding over-fitting of the model to the samples.

We utilize the human-robot hand grip data of the study described in Section 4, and collect the temporal sequences of tactile sensing readings from 11 (non-hand) objects during hand grip execution, storing both the raw value of the magnetic flux (Oersted, Oe) and the force (N) estimated from the magnetic flux. On each object the robot executed the three handshake primitives (resulting in 3 grasps per object) as well as three empty grasps. These grasps form the "no hand" class. The selected objects are shown in Figure 6 , which cover both rigid $(0,4)$ and deformable objects $(1,2,3,5,6,7,8,9)$. It is also worth mentioning that the used objects do not have magnetic properties, which could influence the results due to the principles behind the functioning of the used sensors. Data is then split into training and test sets. We randomly sample 49 handshakes and 26 object grasps for training ( $80 \%$ of the initial dataset) and 11 handshakes and 8 object grasps for testing (remaining $20 \%$ of the dataset). Additionally, we included the "weak" 
Table 3: Handshake classification results for the Field Based Classifier on each test run, and the overall miss-classification error mean.

\begin{tabular}{c|cccccccccccccccc}
\hline Iteration & $\mathbf{1}$ & $\mathbf{2}$ & $\mathbf{3}$ & $\mathbf{4}$ & $\mathbf{5}$ & $\mathbf{6}$ & $\mathbf{7}$ & $\mathbf{8}$ & $\mathbf{9}$ & $\mathbf{1 0}$ & $\mathbf{1 1}$ & $\mathbf{1 2}$ & $\mathbf{1 3}$ & $\mathbf{1 4}$ & $\mathbf{1 5}$ & Mean \\
\hline Optimal K & 1 & 1 & 3 & 1 & 1 & 3 & 1 & 1 & 1 & 3 & 1 & 1 & 3 & 5 & 3 & - \\
\hline Miss-class. error & 0.1579 & 0 & 0.1053 & 0 & 0 & 0 & 0.0526 & 0 & 0 & 0 & 0.0526 & 0 & 0 & 0.16 & 0 & $\mathbf{0 . 0 3 5 1}$ \\
\hline
\end{tabular}

Table 4: Handshake classification results for the Force Based Classifier on each test run, and the overall miss-classification error mean.

\begin{tabular}{c|cccccccccccccccc}
\hline Iteration & $\mathbf{1}$ & $\mathbf{2}$ & $\mathbf{3}$ & $\mathbf{4}$ & $\mathbf{5}$ & $\mathbf{6}$ & $\mathbf{7}$ & $\mathbf{8}$ & $\mathbf{9}$ & $\mathbf{1 0}$ & $\mathbf{1 1}$ & $\mathbf{1 2}$ & $\mathbf{1 3}$ & $\mathbf{1 4}$ & $\mathbf{1 5}$ & Mean \\
\hline Optimal K & 11 & 11 & 21 & 13 & 23 & 17 & 13 & 13 & 13 & 13 & 11 & 13 & 9 & 1 & 11 & - \\
\hline Miss-class. error & 0.21 & 0.31 & 0.16 & 0.16 & 0.16 & 0.26 & 0.32 & 0.16 & 0.16 & 0.21 & 0.21 & 0.05 & 0.1 & 0.1 & 0.19 & $\mathbf{0 . 1 9}$ \\
\hline
\end{tabular}

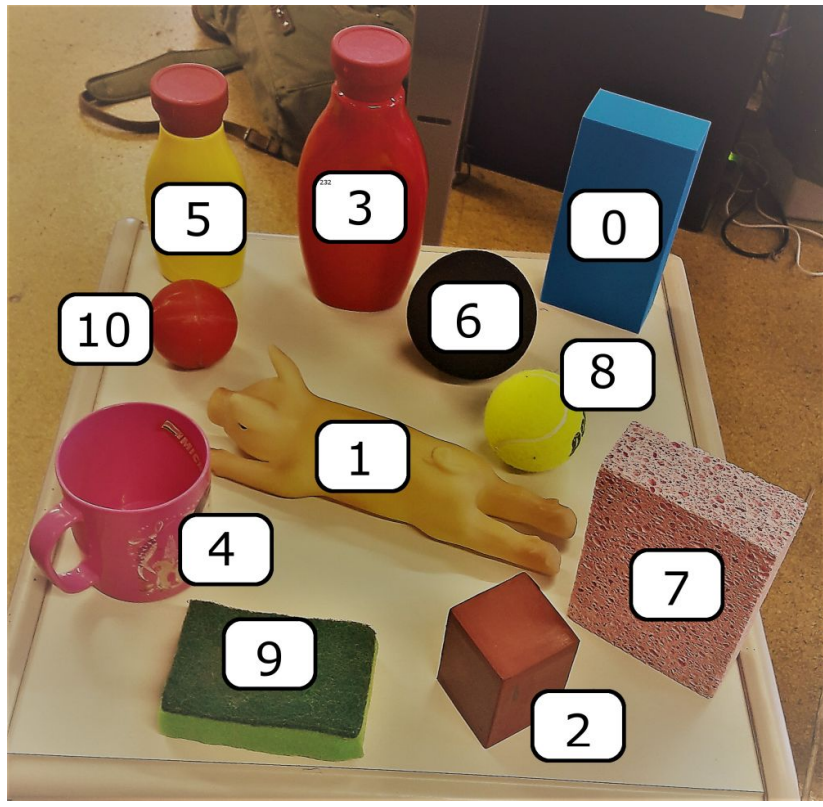

Figure 6: Objects used as the "no hand" class.

and "strong" empty handshakes in the training set and the medium empty handshake in the test set. The reason for this is to have training data for the special cases where the sensors are not in contact with anything.

\subsection{Hyper-parameter tuning and cross-validation}

To tune the hyper-parameter $K$, we perform 7 -fold crossvalidation and choose the $K$ (on a set from 1 to 27 , where $K$ is odd) that yields the lowest average miss-classification error. We use 7 folds because it is the greatest common divisor of the number of handshakes (49) and the number of "no hand" events (28) for training, allowing us to have easy splits.

\subsection{Features and metric}

During our experiments we used two different classifiers: one based on Forces (N) and another based on Fields (Oe). For both classifiers the features are the time series of the three $X, Y, Z$, Cartesian components of the force or magnetic flux, on each of the eleven sensors.

Since each experiment had a different duration, which is not controlled, we used the Dynamic Time Warping [26] algorithm to compute a comparison metric between samples.

\subsection{Results}

Given the small size of the dataset, in order to appropriately test our method we performed 15 iterations of the following procedure:

1. Randomly split the full dataset into training and test sets as described in Subsection 5.2.

2. Perform the 7-fold cross-validation on the training set to find the optimal value of the $\mathrm{K}$ parameter.

3. Test the classifier trained on the full training set on the test set using the optimal $\mathrm{K}$.

The resulting values of the evaluation are summarized in Table 3 and 4. We can see that both classifiers yield inspiring results, with the Field Based Classifier outperforming the Force Based Classifier. We can infer from these results that the Field Based Classifier failed 3 of 19 test items on the worst scenario and the Force Based Classifier failed 6 out of 19 test items on the worst case scenario. 


\section{Human-robot handshake study: customized handshakes}

\subsection{Objectives}

The main objective of this study is to find the hand grip reference force distribution for control, using the feedback from the participant to set the position of the motors that provide better comfort and hand grip strength. The customized hand grips should provide a more accurate distribution of the forces over the sensors than the method described in Section 4. A more accurate force distribution provides sensor data to be used as control reference for a future hand grip controller and gather user feedback for possible improvements regarding the mechanical properties of the hand.

\subsection{User statistics}

We performed these experiments during an exhibition in bachelor's admission day, an event that allowed us to get more participants. The experiment had a total of 35 participants, 28 male and 7 female, with ages between 12 and 49 $(\mu=25.38, \sigma=9.69)$.

\subsection{Methodology}

Taking into account the feedback received in the experiment of Section 4 regarding the robot's palm and the absence of one finger, we designed a new experiment to address:

- Limitations of the three pre-configured tasks derived from the high variability of the subjects hand shape and size. We expect to have a larger force average total, because the finger's positions will fit better with each participant's hand.

- Improve the comfort of the hand grip by performing an iterative selection of the position of the fingers that uses participants' feedback.
Table 5: Average sum of sensor forces of user choice.

\begin{tabular}{c|c}
\hline & Force (N) \\
\hline Female & 15.34 \\
Male & 16.07 \\
Total & 15.92 \\
\hline
\end{tabular}

Table 6: Statistical tests used to compare the "best" predefined handshake from Section 4 and the customized handshake from Section 6. Means and variances plotted on Figure 7.

\begin{tabular}{|c|c|c|}
\hline \multicolumn{3}{|c|}{ Independent samples t-test } \\
\hline Sensor & $t$ & $p$ \\
\hline 1 & 3.13 & 0.003 \\
\hline 2 & 2.03 & 0.047 \\
\hline 4 & 0.452 & 0.653 \\
\hline 5 & 3.680 & 0.001 \\
\hline 6 & 1.755 & 0.085 \\
\hline 8 & 6.303 & $<0.001$ \\
\hline 9 & -8.626 & $<0.001$ \\
\hline \multicolumn{3}{|c|}{ Mann-Whitney U test } \\
\hline Sensor & $U$ & $p$ \\
\hline 3 & 315 & 0.654 \\
\hline 7 & 212 & 0.022 \\
\hline 10 & 134 & $<0.001$ \\
\hline 11 & 11 & $<0.001$ \\
\hline
\end{tabular}

The experiment begins with the human subject receiving a standard hand grip. Then this hand grip is customized by the subject, by increasing or decreasing the force applied by each of the robots fingers, and he/she chooses his or her own preferred handshake grip. This process occurs through the use of a Graphical User Interface (GUI) that controls the position of the fingers. Since the subjects can not be able to use this GUI themselves since they are shaking hands with the robot, they verbally instruct a researcher that controls the GUI on how to move the robot's fingers. During the finger position adjustment, we record the motor encoder position of each finger for the selected handshake grip. This customized handshake is then performed again on the subject while recording the forces on each tactile sensor, and we ask the participant for a confirmation if the handshake is comfortable.

From suggestions given by the subjects of the experiment in Section 4 and to increase the comfort of the robot 


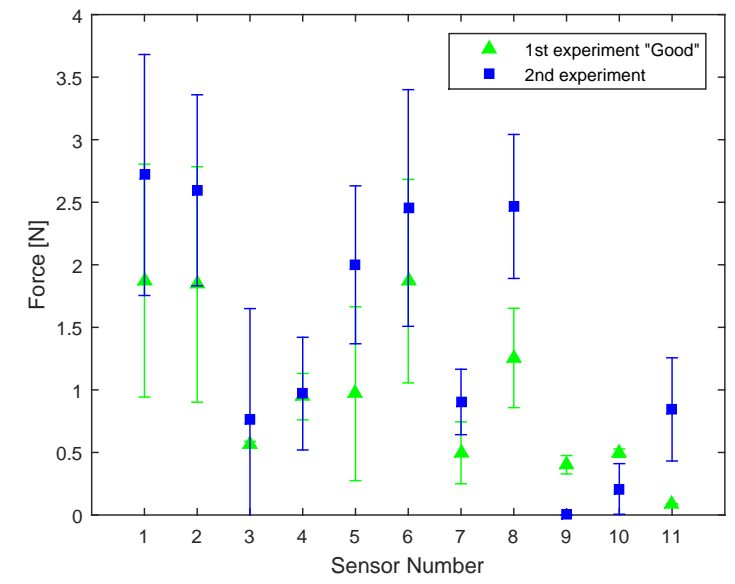

Figure 7: Average and variance of the force measured on each sensor for the ideal hand grip chosen by the subjects (blue), and the average of the hand grip evaluated as "good" (green) in the experiment of Section 4 (Figure 5).

hand, the experiments were performed with the robot fitted with a 3D printed palm that can be seen in Figure 3.

\subsection{Results and discussion}

Like in the first experiment we present the average total force magnitude chosen by the subjects for each sensor. In this case, there is only one value for each sensor since the experiment has only one final position of handshake (the optimal one). The results are presented in Figure 7. We notice that sensors 1, 2, 5, 6 and 8 are the ones which perform a larger contact with the hand of the subject, while some of them almost make no contact. Comparing to the first experiment, we see larger forces applied practically in all sensors that result in a larger overall force applied, as presented in Table 5 and Figure 7. Comparison of force sample distributions on each sensor for both hand grasps shows statistically significant differences for the majority of sensors (Sensors 1, 2, 5, 7, 8, 9, 10 and 11 - 8 out of 11 $72.7 \%$ ) on both conditions. In addition, during the second execution of the handshake, all the users confirm that the selected handshake is comfortable.

\subsection{Qualitative feedback}

Overall the users enjoyed the handshake with the robot. They also reported that they were not expecting the sensors to be as comfortable as they were. The palm of the robot was also well rated, although some users said that having the palm with the same sensors would provide an even more comfortable handshake with the added advantages of increasing the tactile data, a fact that would increase the perceived safety.

\section{Force control hand grip user study}

In this Section we present a study where the participants evaluate two handshakes: one where the hand grip has fixed joint positions set to be the mean positions resulting from the experiment on Section 6 (which we will refer to as "Fixed HS") and other with force control described in Subsection 7.1 (which we will refer to as "PID HS") The set point of the force controller is obtained from the average force per sensor for all people from the study in Section 6 .

The study had a within-subjects design, with each participant being exposed to both handshakes. The handshake order was counterbalanced to avoid possible biases. After each handshake the participant evaluated it in a questionnaire that considers Perceived Enjoyment (PE) questions [27], Interaction (INT) questions [28], safety during interaction $[29,30]$, and handshake firmness and strength. Participants had no prior knowledge of technical details of each handshake or what differed between them.

\subsection{Force control approach}

Each finger of the robot poses a multiple input single output system, where the multiple inputs are the force sensor values and the output is the motor encoder position. However, the physical model of an underactuated finger that opens and closes is not very accurate, since the motion of the limbs and the forces applied on the sensors depend on several variables such as the initial point of contact with the object, the object's shape and other physical properties such as roughness and elasticity. Thus, our approach for control is a PID controller [31]. Our controller has as process variable, the $\ell^{2}$ norm of the forces measured on the sensors for each finger as follows:

$$
\begin{array}{r}
F_{s_{i}}=\sqrt{F_{\chi, s_{i}}^{2}+F_{y, s_{i}}^{2}+F_{z, s_{i}}^{2}}, \\
F_{f_{j}}=\sum_{s_{i}} F_{s_{i}}, \forall s_{i} \in f_{j},
\end{array}
$$

where $s_{i}$ stands for sensor $i$ and $f_{j}$ stands for finger $j$. In Eq. (1), $F_{s_{i}}$ is the magnitude of the force on sensor $s_{i}$, and in Eq. (2) $F_{f_{j}}$ is the sum of the sensors mounted on the finger $f_{j} . F_{f_{j}}$ is the process variable of the controller, and the set value is obtained from the mean value of the forces $F_{X, s_{i}}, F_{y, s_{i}}$ and 
$F_{z, s_{i}}$ for every sensor across all the users of the customized handshake study in Section 6.

\subsection{Robot's arm shake motion}

A realistic human-robot handshake should control autonomously the arm and hand motor joints using force and torque feedback. On one hand, we are able to define a closed-loop force control using the finger sensors to control the motors of the fingers. On the other hand, our robot's sensing capabilities of the arm joints are limited to closed-loop position and velocity control. Having this limitation in mind, we implemented an arm motion inspired on the human-human handshake model proposed by [32]. In Figure 8, we show the evolution of the position of the robot's wrist during the handshake. Note that we control the elbow and wrist joints together to keep the same robot's hand orientation while the robot is performing the arm motion. The handshakes executed by the robot are composed by the arm shake trajectory of this section and the hand grips under study ("Fixed HS" or "PID HS").

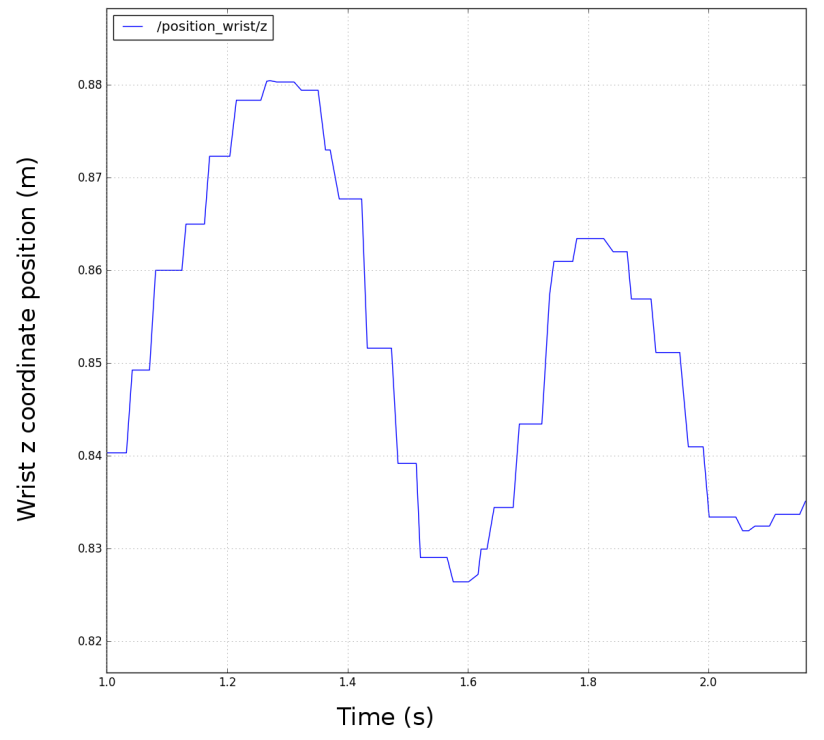

Figure 8: Predefined arm motion to perform the handshake. but did not have robotics or electrical and computer engineering background nor did they have any prior knowledge about the experiment, thus avoiding possible biases.

The study was performed as follows. First a researcher would present the robot and assign a code to the participant: " $\mathrm{A}$ " or "B". The purpose of these codes was to let the researcher controlling to robot know which handshake to perform first: "A" - "Fixed HS" followed by "PID HS" and "B" - "PID HS" first followed by "Fixed HS". Then the participant was instructed to shake hands with the robot and answer the questionnaire shown in Table 7. Afterwards the participant would be instructed to shake hands with the robot again (which was the second handshake), and answer the questionnaire about the second handshake. With this questionnaire we intended to evaluate how people perceive the interaction with the handshakes (with INT items [28]), handshake firmness (FRM), strength (SRT) and handshake safety (SFT) [29, 30]. Finally PE items reported on people's Perceived Enjoyment [27].

Table 7: Questionnaire used to evaluate and compare hand grips.

\begin{tabular}{l|l}
\hline Code & \multicolumn{1}{c}{ Item } \\
\hline & $\begin{array}{l}\text { How did you feel about you interaction with } \\
\text { the robot? }\end{array}$ \\
INT1 & Scary (1) - Not scary (7) \\
INT2 & Boring (1) - Interesting (7) \\
INT3 & Meaningless (1) - Meaningful (7) \\
INT4 & Unexciting (1) - Exciting (7) \\
\hline FRM & The handshake was firm \\
& Totally disagree (1) - Totally agree (7) \\
\hline STR & I think the handshake was: \\
& Too weak (1) - Too strong (7) \\
\hline & During the handshake I was feeling: \\
SF1 & Anxious (1) - Relaxed (7) \\
SF2 & Agitated (1) - Calm (7) \\
SF3 & Surprised (1) - Quiescent (7) \\
\hline PE1 & I enjoy the robot's handshake \\
PE2 & I find it fun to handshake the robot \\
PE3 & I find the handshake pleasurable \\
& Totally disagree (1) - Totally agree (7) \\
\hline
\end{tabular}

\subsection{Procedure}

For this experiment we recruited 38 participants, 20 female and 18 male, with ages ranging from 19 to 52 years old ( $\mu=25.39, \sigma=6.34)$. The recruited participants belonged to the academic community (students and staff),

\subsection{Results}

We now analyze the results and compare both hand grips. Since participants went through both conditions we per- 
formed Dependent T-tests to compare results represented by normal data. For non-normal data we used Wilcoxon tests.

\subsubsection{Interaction items}

According to the Shapiro-Wilk test, answers regarding the interaction items do not follow a normal distribution. Thus we compare both conditions using the Wilcoxon test. No statiscally significant differences were found for any item: INT1 $-Z=-0.176, p=0.860$, INT2 $-Z=-1.117, p=$ 0.264 , INT3 $-Z=-0.730, p=0.466$, INT4 $-Z=-0.192$, $p=0.848$ (Figure 9 and Figure 10). During both conditions the interaction with the robot was not considered scary ( $p<0.001)$, and was considered interesting $(p<0.001)$, meaningful $(p<0.001)$ and exciting $(p<0.001)$ when comparing the median values of these items with the neutral value using a Wilcoxon Signed Rank test.

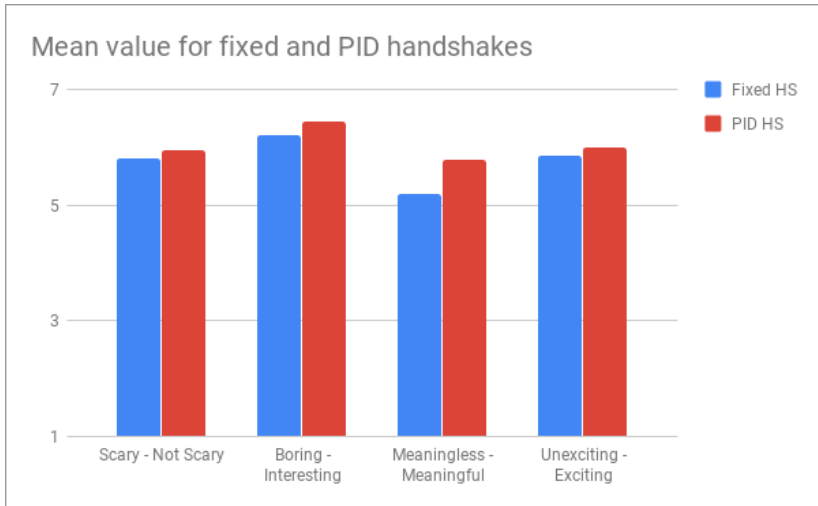

Figure 9: Mean values for interaction items.

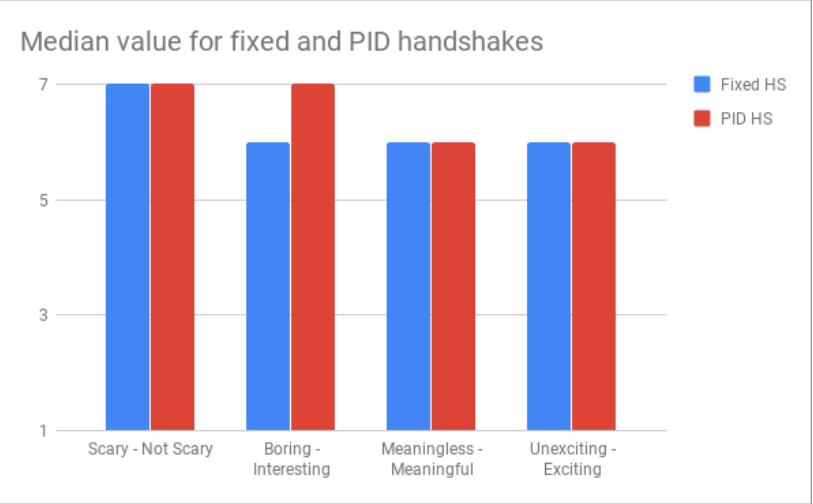

Figure 10: Median values for interaction items.

\subsubsection{Firmness and strength items}

A Shapiro-Wilk test on the firmness (FRM) and strength (STR) items rejected the hypothesis for normal data, which led us to compare the results with the Wilcoxon test. This test yielded no statistically significant differences between conditions. We found a small tendency to find the "Fixed HS" hand grip firmer $\left(M d n_{\text {fixed }}=6.0\right.$ and $M d n_{P I D}=$ 5.5). Regarding the strength, both conditions had a median value of 4, which is the ideal value (Figure 11 and Figure 12). However, a Wilcoxon signed rank test rejects the hypothesis that the true median of both distributions is equal to 4 ( $p<0.05$ for both conditions). Looking at the mean values we can see that they are $\mu_{\text {Fixed }}=4.42$ and $\mu_{\text {Fixed }}=4.34$ meaning that people might have found the grips a bit tighter than the ideal.

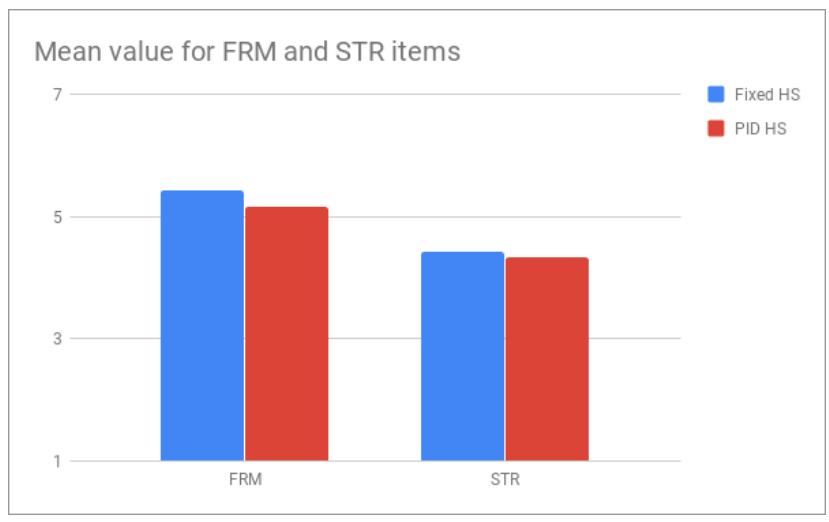

Figure 11: Mean values for firmness and strength items.

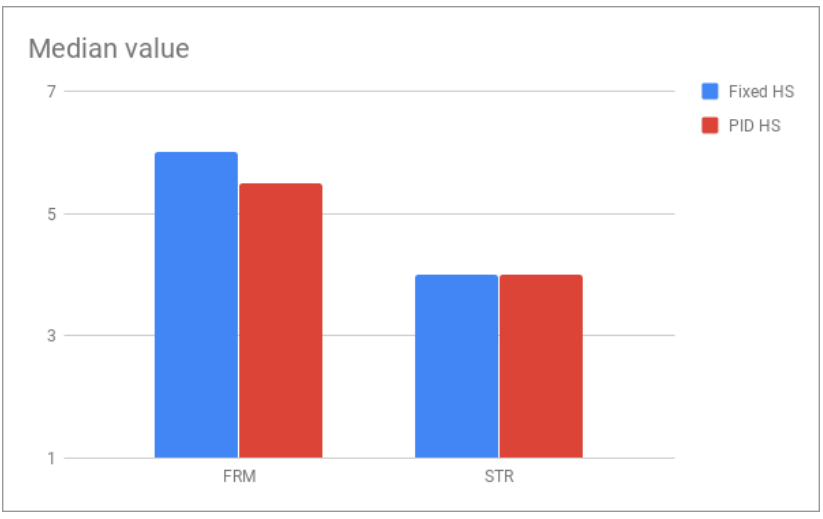

Figure 12: Median values for firmness and strength items. 


\subsubsection{Perceived Safety and Perceived Enjoyment}

The Shapiro-Wilk test could not reject the hypothesis of normal data for the Perceived Safety dimension $(\alpha=$ 0.804). Thus, we compared both conditions using a dependent t-test. No statistically significant differences were found between conditions $(t=-0.9590, p=0.3440)$. We found a very small tendency to perceive the "PID HS" hand grip safer than the "Fixed HS" hand grip: $\mu_{\text {fixed }}=4.9123$, $\sigma_{\text {fixed }}=1.298$, and $\mu_{P I D}=5.1930, \sigma_{\text {fixed }}=1.038$. A OneSample t-test showed that people found both handshakes significantly safe $(t=4.331, p<0.001$ for the "Fixed HS", and $t=7.080, p<0.001$ for the "PID HS").

Normality analysis of the gathered data regarding the Perceived Enjoyment dimension $(\alpha=0.847)$ using the Shapiro-Wilk test rejected the hypothesis of normal data, leading us to compare both handshakes with the Wilcoxon test. No statistically significant differences were found between conditions $(Z=-0.816, p=0.414)$. Nonetheless, data shows a small tendency towards the "PID" version of the hand grip: $M d n_{\text {fixed }}=5.667$, and $M d n_{P I D}=6.0$ (Figure 13 and Figure 14). A Wilcoxon Signed Rank test against the neutral value shows that people enjoyed both handshakes $(Z=672.0, p<0.001$ for "Fixed HS" and $Z=739.0, p<0.001$ for the "PID HS".

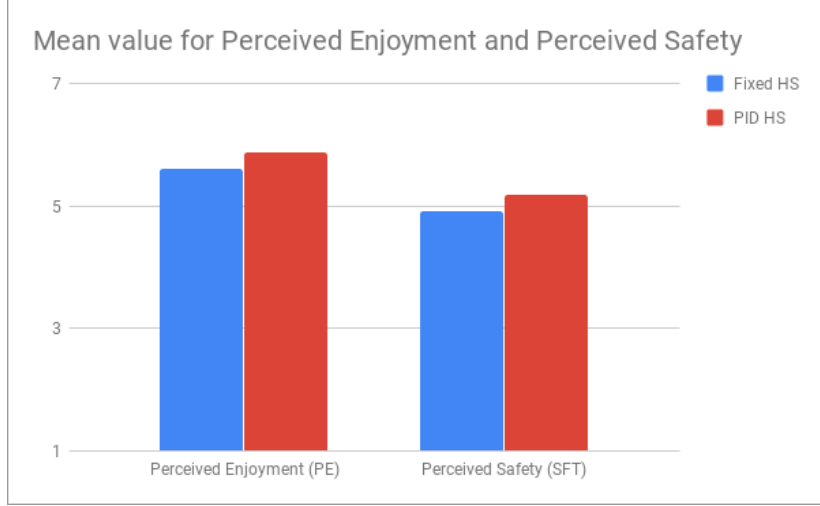

Figure 13: Mean values for Perceived Safety and Perceived Enjoyment.

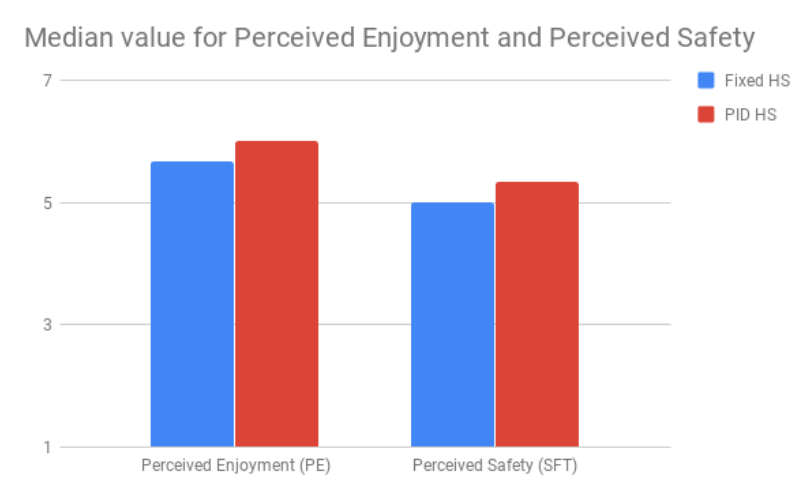

Figure 14: Median values for Perceived Safety and Perceived Enjoyment.

\section{Conclusions and future work}

We developed a platform for exploring human-robot handshaking. By using a novel and state-of-the-art tactile sensor we can accurately measure in real-time the force vector at each contact point.

From the examination of the human subject data of the pilot study in Section 4 we identified initial flaws in the design of the handshake study and initial improvements of the robot's hand, like the hand palm. The limited number of grip trajectories limits the analysis of the user's preferred grip force, since the "optimal" grip strength for that user might not be part of available hand grips. We realized that by using fixed grip motions, the actual contact forces perceived by the users will depend highly on the size and shape of the hand of the human subject. Also, the limited number of grip trajectories limits the analysis of the user's preferred grip force. This issue was addressed in Section 6 , by letting the user control the position of each finger. It is interesting to note that the overall preferred forces readings on each of the sensors was significantly higher in the second experiment. This fact could be due to increased perceived safety and improved comfort provided by the hand with a palm cover. Nonetheless, this is just a hypothesis, since groups in both experiments are different. The sensor forces gathered during the customized hand grip provided us with the set point values for a force-based controller, which was evaluated in the study in Section 7. We compared the force based controller against a finger position based controller where the set points are the average position values obtained on Section 6. No statistically significant differences were found between both approaches in any of the measured items. We could only find small tendencies regarding interaction items towards the "PID HS". Similarly there was a small tendency to find the 
"PID HS" safer and more enjoyable than the "Fixed HS". Both hand grips were positively evaluated by the participants, which leads to the hypothesis that low-end robotic systems can actually perform comfortable handshakes for populations similar to the ones in the present study without the need for touch sensors, by following a methodology similar to the one described in Section 6. Generalization requires further investigation with other robotic platforms and hands. Nonetheless, using a "PID HS" hand grip has the major benefit of improving safety, even if no statistically significant differences were found in people's perceptions. We will further apply Reinforcement Learning methods to make the robot autonomously explore and adapt to the contact forces, without restricting to a set of predefined handshakes, to give the most natural and comfortable handshake for humans according to the user feedback. The qualitative feedback received from the users (Subsections 4.6 and 6.5) allowed us to pinpoint several problems in the mechanical design of the hand and sensors regarding user comfort and the mechanical robustness of the sensors. With this insights in mind we intend to improve the robot's hand in this matter. However, people's preferences regarding hand grip forces and their distributions seem to be influenced by personality measures [33], making it difficult to have a single grip reference (even in terms of force control) that suits everybody. Although the gathered data does not currently allow customized handshakes for each individual (in regards of applied force), we hypothesize that we might be able to learn and predict individual preferences with the future implementation of a tactile palm that has an array of tactile sensors plus biosignals such as temperature and sweating sensors. This might allow us to measure the forces exerted by the person on the robot's hand, which might be correlated to the preferable force distribution for that person. We intend to analyse feasibility of this approach in future works. The addition of extra sensors should also provide an improvement of the measurements of force distributions along the hand of the robot, which in turn will reduce uncomfortable grasps due to exceeding force on an unmeasured area. We also intend to develop other control methods using extra sensors and evaluate if the resulting controller is a significant improvement over the suggested control methods presented on this work.

In the handshake detection study, we achieved promising results with both handshake classifiers, being able to correctly classify the majority of the "hand" and "no-hand" events. Overall the Field Based classifier showed better performance than the Force Based classifier, but does not generalize (like the Force Based classifier does) to other systems since the magnetic fields depend on each individual sensor's construction. In future work we intend to make use of all sensors in all four fingers of the robot and extra palm sensors, with a larger dataset (and with more object variability) to further analyse the performance of the classifiers.

Our work so far has only focused on the force applied to the contacts during the handshake. In order to obtain a natural interaction, we will need to make the robot's arm move in a human-like way. Previous works cannot be applied in a straightforward way to our platform since it makes use of the forces measured in each robot joint to synchronize with the human motion, i.e. Neural Oscillators of Kasuga et al. [6], the shake-motion leading model of Yamato et al. [9] and the models proposed by Avraham et al. [16]. Our robot does not currently report the torques applied to each joint but provides very detailed information about the forces on the contacts of the hand. We are exploring other approaches to arm motion generation that may exploit the available tactile force perception, e.g. movement primitives for force interaction. A Turing-like handshake test [16] adapted for the full handshake (personalized grip strength and arm motion) should be a good benchmark for future handshake systems with those functionalities.

Although the protocols of the experiments in Sections 6 and 7 were just tested on Vizzy, these protocols can be repeated and applied in many different robots. If the robot has no force control possibilities, one can use the protocol of Section 6 to get the mean value of joint positions. Otherwise, the protocol of Section 6 can be used to extract a reference force distribution for hand grip control using the control method of Section 7. If the robot's hand is anthropomorphic with similar dimensions, sensing and degrees of freedom, the forces obtained in this study are transferable to other hands.

Acknowledgement: Many thanks to Rui Miguel Figueiredo for his help during the handshake experiments presented in Section 7. This work was supported by the Portuguese Fundação para a Ciência e a Tecnologia [UID/EEA/50009/2013] and [SFRH/BD/133098/2017], and FCT project AHA [CMUP-ERI/HCI/0046/2013].

\section{References}

[1] T. Paulino, P. Ribeiro, M. Neto, S. Cardoso, A. Schmitz, J. SantosVictor, et al., Low-cost 3-axis soft tactile sensors for the humanfriendly robot vizzy, In: 2017 IEEE International Conference on Robotics and Automation (ICRA 2017), 2017, 966-971 
[2] A. Chortos, J. Liu, Z. Bao, Pursuing prosthetic electronic skin, Nature Materials, 2016, 15(9), 937-950

[3] A. De Santis, B. Siciliano, A. De Luca, A. Bicchi, An atlas of physical human-robot interaction, Mechanism and Machine Theory, 2008, 43(3), 253-270

[4] Y. S. Sefidgar, K. E. MacLean, S. Yohanan, H. M. Van der Loos, E. A. Croft, E. J. Garland, Design and evaluation of a touchcentered calming interaction with a social robot, IEEE Transactions on Affective Computing, 2016, 7(2), 108-121

[5] S. Soyguder, T. Abut, Haptic industrial robot control with variable time delayed bilateral teleoperation, Industrial Robot: An International Journal, 2016, 43(4), 390-402

[6] T. Kasuga M. Hashimoto, Human-robot handshaking using neural oscillators, In: 2005 IEEE International Conference on Robotics and Automation (ICRA 2005), 2005, 3802-3807

[7] D. Papageorgiou Z. Doulgeri, A kinematic controller for humanrobot handshaking using internal motion adaptation, In: 2015 IEEE International Conference on Robotics and Automation (ICRA 2015), 2015, 5622-5627

[8] K. Ouchi S. Hashimoto, Handshake telephone system to communicate with voice and force, In: 6th IEEE International Workshop on Robot and Human Communication, RO-MAN'97 SENDAI, 1997, 466-471

[9] Y. Yamato, M. Jindai, T. Watanabe, Development of a shakemotion leading model for human-robot handshaking, In: 2008 SICE Annual Conference, 2008, 502-507

[10] M. Jindai T. Watanabe, Development of a handshake robot system based on a handshake approaching motion model, In: 2007 IEEE/ASME International Conference on Advanced Intelligent Mechatronics, 2007, 1-6

[11] M. Jindai, S. Ota, Y. Ikemoto, T. Sasaki, Handshake request motion model with an approaching human for a handshake robot system, In: 2015 IEEE 7th International Conference on Cybernetics and Intelligent Systems (CIS) and IEEE Conference on Robotics, Automation and Mechatronics (RAM), 2015, 265-270

[12] M. Jindai T. Watanabe, Development of a handshake request motion model based on analysis of handshake motion between humans, In: 2011 IEEE/ASME International Conference on Advanced Intelligent Mechatronics (AIM), 2011, 560-565

[13] S. Ota, M. Jindai, T. Fukuta, T. Watanabe, A handshake response motion model during active approach to a human, In: 2014 IEEE/SICE International Symposium on System Integration, 2014, 310-315

[14] S. Ota, M. Jindai, T. Sasaki, Y. Ikemoto, Handshake response motion model with approaching of human based on an analysis of human handshake motions, In: 2015 7th International Congress on Ultra Modern Telecommunications and Control Systems and Workshops (ICUMT), 2015, 8-13

[15] A. Melnyk, P. Henaff, V. Khomenko, V. Borysenko, Sensor network architecture to measure characteristics of a handshake between humans, In: 2014 IEEE 34th International Scientific Conference on Electronics and Nanotechnology (ELNANO), 2014, 264-268

[16] G. Avraham, I. Nisky, H. L. Fernandes, D. E. Acuna, K. P. Kording, G. E. Loeb, et al., Toward perceiving robots as humans: Three handshake models face the turing-like handshake test, IEEE Transactions on Haptics, Third 2012, 5(3), 196-207, ISSN 1939-1412

[17] N. Pedemonte, T. Laliberté, C. Gosselin, Design, control, and experimental validation of a handshaking reactive robotic inter- face, Journal of Mechanisms and Robotics, 2016, 8(1), 011020

[18] E. Knoop, M. Bächer, P. Beardsley, Contact pressure distribution as an evaluation metric for human-robot hand interactions, In: HRI 2017 workshop - Towards reproducible HRI Experiments: Scientific endeavors, benchmarking and standardization, 2017

[19] P. Orefice, M. Ammi, M. Hafez, A. Tapus, Let's handshake and i'll know who you are: Gender and personality discrimination in human-human and human-robot handshaking interaction, In: 2016 IEEE-RAS 16th International Conference on Humanoid Robots (Humanoids), 2016, 958-965

[20] D. S. Chathuranga, Z. Wang, Y. Noh, T. Nanayakkara, S. Hirai, Robust real time material classification algorithm using soft three axis tactile sensor: Evaluation of the algorithm, In: 2015 IEEE/RSJ International Conference on Intelligent Robots and Systems (IROS), 2015, 2093-2098

[21] S. Luo, W. Mou, M. Li, K. Althoefer, H. Liu, Rotation and translation invariant object recognition with a tactile sensor, In: SENSORS, 2014 IEEE. IEEE, 2014, 1030-1033

[22] A. Khasnobish, G. Singh, A. Jati, A. Konar, D. Tibarewala, Objectshape recognition and $3 \mathrm{~d}$ reconstruction from tactile sensor images, Medical \& biological engineering \& computing, 2014, 52 (4), 353-362

[23] P. Moreno, R. Nunes, R. Figueiredo, R. Ferreira, A. Bernardino, J. Santos-Victor, et al., Vizzy: A humanoid on wheels for assistive robotics, In: Robot 2015: Second Iberian Robotics Conference, 2016, 17-28

[24] H. Sakoe S. Chiba, Dynamic programming algorithm optimization for spoken word recognition, IEEE transactions on acoustics, speech, and signal processing, 1978, 26(1), 43-49

[25] H. Ding, G. Trajcevski, P. Scheuermann, X. Wang, E. Keogh, Querying and mining of time series data: experimental comparison of representations and distance measures, Proceedings of the VLDB Endowment, 2008, 1(2), 1542-1552

[26] D. J. Berndt J. Clifford, Using dynamic time warping to find patterns in time series, In: 3rd International Conference on Knowledge Discovery and Data Mining, AAAIWS'94, 1994, 359-370

[27] H. Sun P. Zhang, Causal relationships between perceived enjoyment and perceived ease of use: An alternative approach, Journal of the Association for Information Systems, 2006, 7(9), 24

[28] W. A. Bainbridge, S. Nozawa, R. Ueda, K. Okada, M. Inaba, A methodological outline and utility assessment of sensor-based biosignal measurement in human-robot interaction, International Journal of Social Robotics, Aug 2012, 4(3), 303-316

[29] D. Kulić E. Croft, Physiological and subjective responses to articulated robot motion, Robotica, 2007, 25(1), 13-27, https://doi.org/10.1017/S0263574706002955

[30] C. Bartneck, D. Kulić, E. Croft, S. Zoghbi, Measurement instruments for the anthropomorphism, animacy, likeability, perceived intelligence, and perceived safety of robots, International Journal of Social Robotics, Jan 2009, 1(1), 71-81, https://doi.org/10.1007/s12369-008-0001-3

[31] J. G. Ziegler N. B. Nichols, Optimum settings for automatic controllers, Journal of Dynamic Systems, Measurement, and Control, 1942, 115(2B), 220-222

[32] Z. Wang, J. Yuan, M. Buss, Modelling of human haptic skill: a framework and preliminary results, Proceedings of the 17 th IFAC World Congress, 2008, 41(2), 14761-14766

[33] W. F. Chaplin, J. B. Phillips, J. D. Brown, N. R. Clanton, J. L. Stein, Handshaking, gender, personality, and first impressions., Journal of personality and social psychology, 2000, 79(1), 110 